\title{
Gastrostomia e jejunostomiar aspectos da evolução técnica e da ampliação das indicações
}

\section{Gastrostomy and jejunostomy: aspects of the technical evolution and widening of indications}

José Sebastião dos Santos ${ }^{1}$, Rafael Kemp², Ajith Kumar Sankarankutty ${ }^{1}$, Wilson Salgado Junior ${ }^{1}$, Luiz Fernando Tirapelli ${ }^{3}$, Orlando de Castro e Silva Júnior ${ }^{1}$

\begin{abstract}
RESUMO
O acesso à luz do estômago e do jejuno proximal por meio de gastrostomia e jejunostomia, respectivamente, de forma temporária ou definitiva, está indicado diante da necessidade prolongada de descompressão digestiva ou de suporte alimentar. $\mathrm{O}$ emprego desses procedimentos expandiu-se nos últimos 25 anos com a introdução da gastrostomia endoscópica, especialmente em pacientes com afecções neurológicas de evolução progressiva e neoplasias avançadas. Este artigo aborda aspectos conceituais da gastrostomia e jejunostomia, as principais indicações, as vias de acesso preferenciais em diferentes cenários clínicos e as modalidades técnicas frequentemente empregadas. O manejo dessas estomias, os resultados e as potenciais complicações também são enfatizados. Finalmente, os fundamentos éticos e legais da ampliação da indicação da gastrostomia e da jejunostomia como procedimentos paliativos são discutidos.
\end{abstract}

Palavras-chave: Gastrostomia. Gastrostomia Endoscópica. Jejunostomia. Nutrição Enteral. Descompressão Digestiva. cuidados paliativos.

\section{Introdução}

O acesso à luz do estômago e do intestino delgado alto é frequentemente obtido mediante introdução de sondas por via nasal ou oral. Habitualmente, esses procedimentos são indicados para descompressão do trato digestório e ou suporte alimentar por períodos que não excedem um mês. Diante do prolongamento da necessidade da descompressão digestiva ou do suporte alimentar preconiza-se a realização da gastrostomia: uma alternativa mais vantajosa à sondagem nasogástrica por ser mais confortável, permitir maior mobilidade do paciente, não interferir com a respiração e os mecanismos fisiológicos de limpeza das vias aéreas. Nas circunstâncias em que há impossibilidade de realização ou contra-indicação para gastrostomia, a jejunostomia é uma alternativa a ser empregada.
1Docentes, Divisão de Cirurgia Digestiva do Departamento de Cirurgia e Anatomia da Faculdade de Medicina de Ribeirão Preto - USP.

2 Docente da Divisão de Cirurgia Digestiva do Departamento de Cirurgia e Anatomia da Faculdade de Medicina de Ribeirão Preto - USP e médico do Hospital das Clínicas da FMRP-USP.

3 Docente, Departamento de Cirurgia e Anatomia.
Correspondência: José Sebastião dos Santos. Departamento de Cirurgia e Anatomia da FMRP-USP. Av. Bandeirantes, 3900, 14049-900 - Ribeirão Preto / SP. (email: jsdsanto@fmrp.usp.br)

Artigo recebido em 20/07/2010 Aprovado para publicação em 24/02/2011 


\section{Gastrostomia}

A gastrostomia é um procedimento cirúrgico que estabelece o acesso à luz do estômago através da parede abdominal. As vias de acesso habitualmente empregadas para realização da gastrostomia são: laparotomia ${ }^{1}$, endoscopia ${ }^{2,3}$ e laparoscopia. ${ }^{4} \mathrm{O}$ preparo préoperatório consiste de jejum de 6 a 8 horas, instalação de acesso venoso para infusão de fluídos com eletrólitos, analgésicos e sedativos. A profilaxia da infecção de parede abdominal, sobretudo ao redor da sonda, é feita mediante antibioticoterapia profilática intravenosa com cefalosporina. Em condições excepcionais, onde há carcinomatose peritoneal, infiltração tumoral da parede gástrica e ascite, o acesso à luz do estômago através da parede gástrica e da cavidade abdominal pode ser arriscado. Nessas circunstâncias, se o paciente apresenta náuseas e vômitos incontroláveis, a gastrostomia percutânea transesofágica com acesso cervical é uma alternativa a ser empregada. ${ }^{5,6}$

\subsection{Indicações de gastrostomia}

\subsection{Desconpressão gástrica}

A descompressão gástrica pode ser obtida por meio de gastrostomia temporária, recomendada ocasionalmente, como complemento de operações abdominais de grande porte para as quais se prenuncia estase gástrica, "íleo adinâmico" prolongado e fístulas digestivas. O procedimento é indicado em pacientes com doença pulmonar obstrutiva crônica, psicóticos, agitados e idosos onde se deseja evitar o desconforto e os riscos do emprego de sonda nasogástrica. Como exemplo, a gastrostomia pode ser realizada em complemento à duodenopancreatectomia cefálica com preservação do piloro. Trata-se de operação de grande porte, habitualmente, empregada para tratamento das neoplasias ampolares e periampolares, e que cursa com retardo do esvaziamento gástrico em 20 a $40 \%$ dos pacientes. ${ }^{7,8}$ Por outro lado, essas taxas na nossa prática clínico-cirúrgica têm se reduzido; em 22 casos consecutivos tratados com essa operação, não realizamos gastrostomia e observamos estase gástrica em apenas um caso $(4,5 \%)$, onde o tratamento foi feito mediante descompressão com sonda nasogástrica e suporte nutricional parenteral por 12 dias.

\subsection{Alimentação}

Temporária: Indicada quando o acesso ao trato digestório está temporariamente prejudicado para recuperação e manutenção do estado nutricional: estenose cáustica, câncer do esôfago e megaesôfago e, eventualmente, no coma prolongado.

Definitiva: como terapêutica paliativa em pacientes portadores de neoplasia maligna irressecável da faringe e do esôfago, para os quais não há condições favoráveis de intubação transtumoral, por via endoscópica ou cirúrgica. As doenças neurológicas: demência, esclerose amiotrófica lateral, seqüelas de acidente vascular cerebral, doença de Parkinson e outras que afetam a motilidade da língua, da faringe e do esôfago e comprometem a deglutição e o apetite também representam um grupo com indicação cada vez mais freqüente de gastrostomia definitiva.

\subsection{Descompressão e alimentação}

Na dependência da evolução pós operatória de algumas cirurgias digestivas mais complexas, a gastrostomia, em determinado momento, pode servir para descompressão do trato digestório e noutra fase ser utilizada para alimentação. No tratamento cirúrgico da obesidade mórbida por meio da operação de derivação gástrica em Y de Roux (cirurgia de Capella), há uma secção do estômago próximo à junção esofagogástrica de forma que a quase totalidade do órgão fica excluída do trânsito. No estômago excluído pode ser realizada a gastrostomia, que, no pós-operatório precoce, servirá para a descompressão gástrica e do trato digestório. A pequena bolsa gástrica remanescente junto ao esôfago será suturada e anastomosada ao jejuno, em Y de Roux. A linha de sutura e a anastomose têm o risco de deiscência, que varia de 1 a $8 \%{ }^{9,10}$ e dessa forma um dreno pode ser deixado no entorno para monitorar eventuais vazamentos. $\mathrm{Na}$ eventualidade de ocorrer uma fístula dirigida para a pele, o dreno abdominal é mantido e resolvido o "íleo adinâmico", inicia-se a dieta pela gastrostomia, na expectativa de fechamento da fístula no período de até seis semanas (Figura. 1).

\subsection{Gastrostomia por laparotomia: aspectos técnicos}

\subsection{Gastrostomia à Stamm ${ }^{11}$ (Figura 2)}

- Incisão: Mediana supra-umbilical;

- Preensão da parede gástrica anterior com pinças de Babcock para avaliar a aproximação do estômago com o peritônio;

- Sutura seromuscular em bolsa a meia-distância entre as curvaturas gástrica maior e menor;

- Incisão com bisturi ou eletrocautério no centro da 


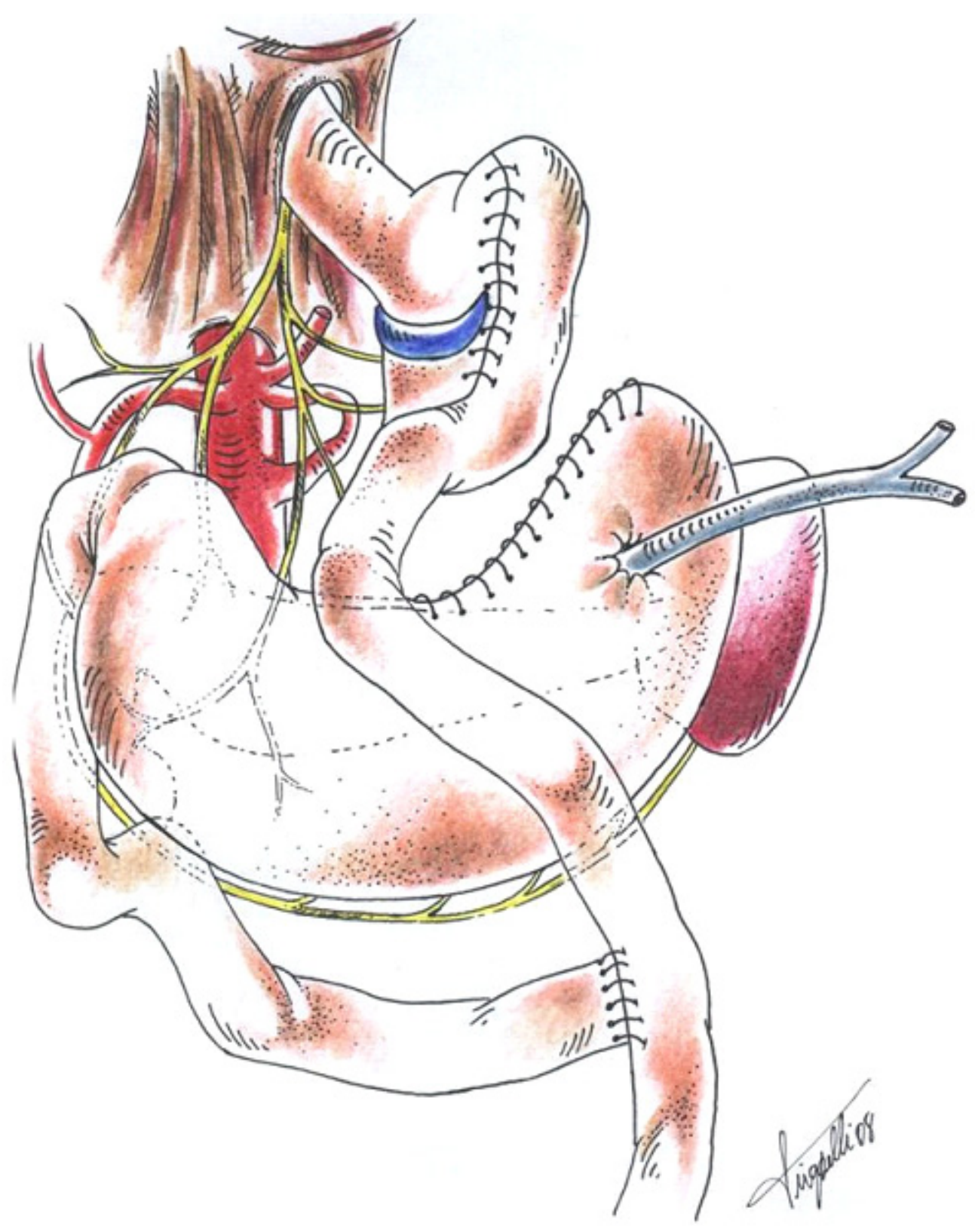

Figura 1: Derivação gástrica em $Y$ de Roux (cirurgia de Capella), com gastrostomia no estômago excluído que tem a finalidade de descomprimir o trato digestório no pós operatório precoce e, eventualmente, servir para alimentar caso haja deiscência na bolsa gástrica com fístula dirigida.

sutura, de tamanho suficiente, para a colocação de sonda com 20 a 26 French;

- Introdução de sonda: Malecot, Pezzer ou Foley, na extensão de 5-6 cm, seguida de fechamento de sutura em bolsa;

- Realização de uma e, eventualmente, duas novas suturas seromusculares sucessivas em bolsa, con- cêntricas, que são amarradas de maneira a cada uma invaginar a anterior;

- Preensão da linha alba com duas pinças de kocher, exposição do hipocôndrio esquerdo e incisão na pele, de tamanho suficiente para passagem da sonda, na parede anterior do abdômen a $2 \mathrm{~cm}$ da reborda costal trans ou pararretal à esquerda; 
- Introdução de uma pinça de Schinidt, a partir da incisão da parede abdominal e preensão da sonda colocada no estômago e um dos fios da sutura em bolsa deixado como reparo para exercer tração do estômago;

- Exteriorização pela contra-abertura do conjunto (fio de reparo e sonda) de forma cuidadosa para evitar a retirada da sonda;

- Tração externa com as duas pinças de kocher utilizadas para preensão da linha alba e contra-tração por meio dos dedos indicadores da parede do hipocôndrio esquerdo para aproximar o peritônio parietal da parede gástrica;

- Fixação da seromuscular do estômago ao peritônio parietal da parede abdominal anterior com pontos cardinais em torno e juntos da sonda;

- Fixação da sonda à pele com ponto de fio inabsorvível;

- Síntese da parede com aproximação da linha alba mediante sutura contínua com fio monofilamentar 1-0 ou 2-0 e da pele com pontos separados de nylon 3-0.

A sonda dirigida para o antro favorece melhor descompressão gástrica; se o objetivo, porém, for a alimentação, é preferível que a extremidade interna da sonda seja dirigida para o fundo gástrico. Embora menos continente que a de localização alta no estô- mago, a gastrostomia realizada na transição do corpo com o antro tem sido indicada para alimentação quando, após esofagectomia, se pretende utilizar o estômago para a reconstrução do trânsito alimentar.

\subsection{Gastrostomia à Witzel}

Os passos técnicos iniciais das gastrostomia à Witzel são semelhantes aos da gastrostomia à Stamm, incluindo a fixação da sonda ao estômago por uma sutura em bolsa. Na seqüência, deita-se a sonda sobre a parede gástrica e faz-se um túnel de $8-10 \mathrm{~cm}$ de extensão mediante sutura seromuscular (contínua ou com pontos separados de fio absorvível ou inabsorvível) recobrindo-a (Figura. 3) e procede-se a exteriorização por contra-abertura. O objetivo desse túnel é a prevenção de vazamento ao redor da sonda exteriorizada na parede abdominal.

A gastrostomias à Stamm e à Witzel, onde os túneis para a colocação de sondas são revestidos de serosa estão indicadas, geralmente, para as necessidades temporárias. Na gastrostomia definitiva, indicada nos transtornos permanentes da deglutição (doenças degenerativas, perda do esôfago cervical, casos crônicos de aspiração incontrolada em algumas formas de paralisia cerebral ou de transtornos laríngeos), as técnicas que criam túnel revestido de mucosa e que, geralmente, prescindem da utilização de sondas, são preferíveis.

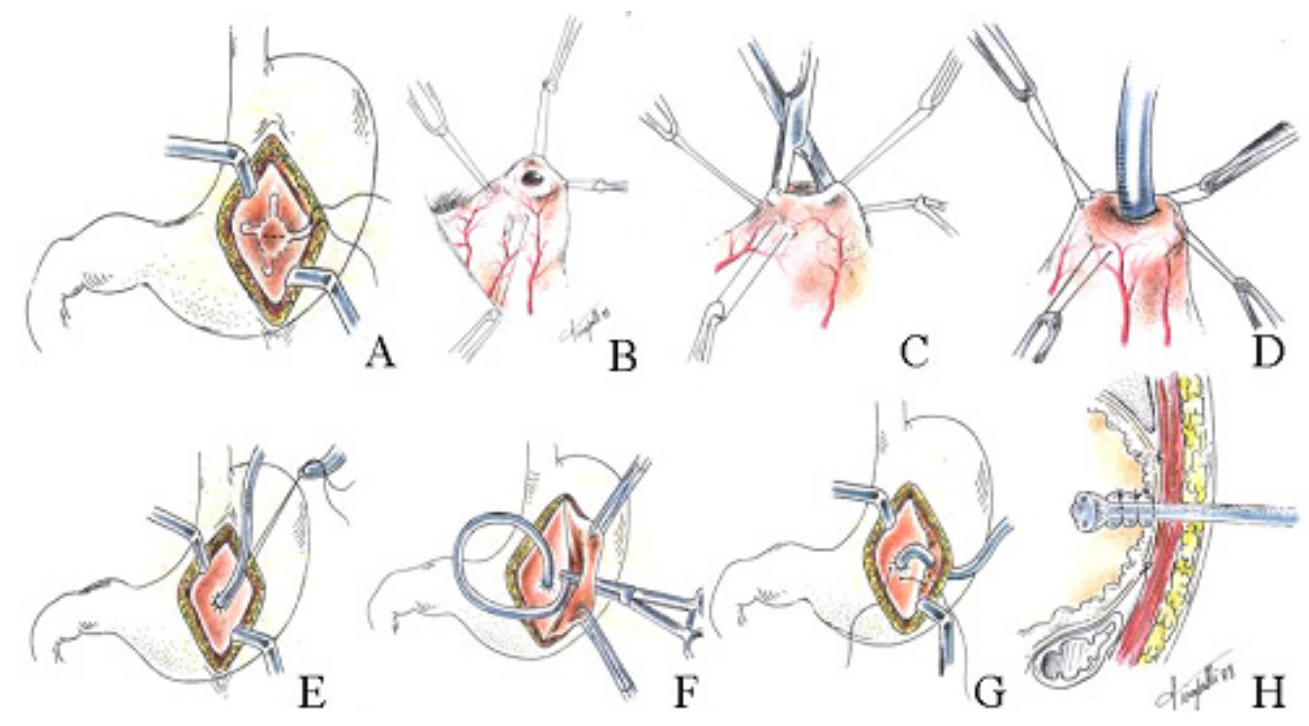

Figura 2: Gastrostomia à Stamm: A) Incisão mediana supra-umbilical e sutura em bolsa na parede gástrica anterior. B) Gastrotomia no centro da sutura em bolsa. C) Certificação do acesso à luz do estômago. D) Introdução da sonda. E) realização de nova sutura em bolsa invaginante. F) Exteriorização da sonda por contra-abertura. F) Início da fixação do peritônio visceral ao parietal. G) Estômago fixo à face interna da parede abdominal com sonda no seu interior e exteriorizada por contra-abertura. 

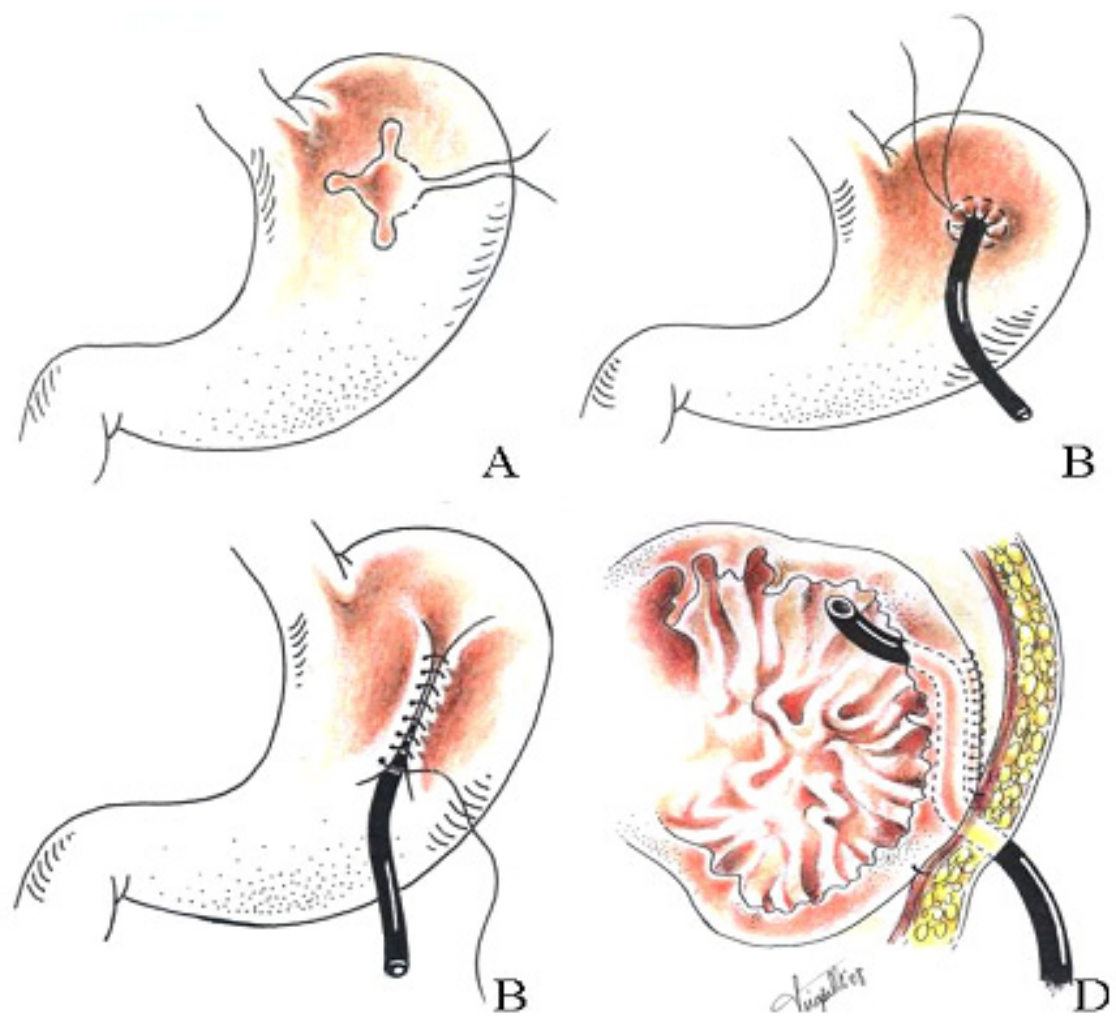

Figura 3: Gastrostomia à Witzel: A) sutura em bolsa. B) introdução da sonda após gastrotomia e realização de nova sutura em bolsa invaginante da mucosa. C) túnel com pontos seromusculares recobrindo a sonda. D) exteriorização da sonda por contra-abertura.

\subsection{Gastrostomia à Depage-Janeway}

A gastrostomia a Depage-Janeway está indicada nas situações de necessidade permanente. Envolve a preparação de um tubo gástrico a partir de um retalho da parede anterior do estômago de aproximadamente 4 a $5 \mathrm{~cm}$, tendo a curvatura maior como base: a extremidade distal do tubo é exteriorizada por contra-abertura na parede abdominal a $3-5 \mathrm{~cm}$ à esquerda da incisão primária e é fixada à pele (Figura. 4). O tubo gástrico pode ser feito mediante emprego do grampeador. ${ }^{1} \mathrm{O}$ manejo dessa modalidade de gastrostomia é mais fácil, a sonda é utilizada apenas durante a passagem do alimento e o estoma pode ser recoberto nos intervalos porque não há risco de fechamento. Por outro lado, os detalhes técnicos são maiores e na prática clínica, a maioria das gastrostomias são realizadas com os fundamentos da técnica de Stamm e o emprego de sondas.

\subsection{Gastrostomia endoscópica percutânea}

O uso prolongado da sonda nasoentérica pode levar à complicações como lesões de asa nasal, sinu- site crônica, refluxo gastroesofágico e pneumonia aspirativa. A gastrostomia cirúrgica ou endoscópica representa uma alternativa para minimizar essas complicações e manter uma via de alimentação enteral de longa duração. $\mathrm{O}$ acesso ao estômago por meio da combinação das vias endoscópica e percutânea foi desenvolvido a partir de $1980^{2}$ e, é considerado um método efetivo em promover nutrição enteral nos pacientes que possuem o trato digestivo funcional, mas por alguma razão, estão impossibilitados de manter ingesta oral adequada. Atualmente, a gastrostomia endoscópica percutânea (GEP) é o método de escolha para nutrição enteral prolongada. As principais indicações desse procedimento estão citadas na tabela abaixo (Tabela 1). ${ }^{12}$

Quando comparado com outros métodos de nutrição enteral, como a alimentação por sonda nasoentérica, a GEP apresenta vantagens em termos de conforto para o paciente, além de menores taxas de sangramento local, obstrução do conduto de alimentação e deslocamento do tubo. Apesar de não impedir o refluxo gastroesofágico ou a aspiração do conteúdo gástrico, na GEP há menor incidência des- 

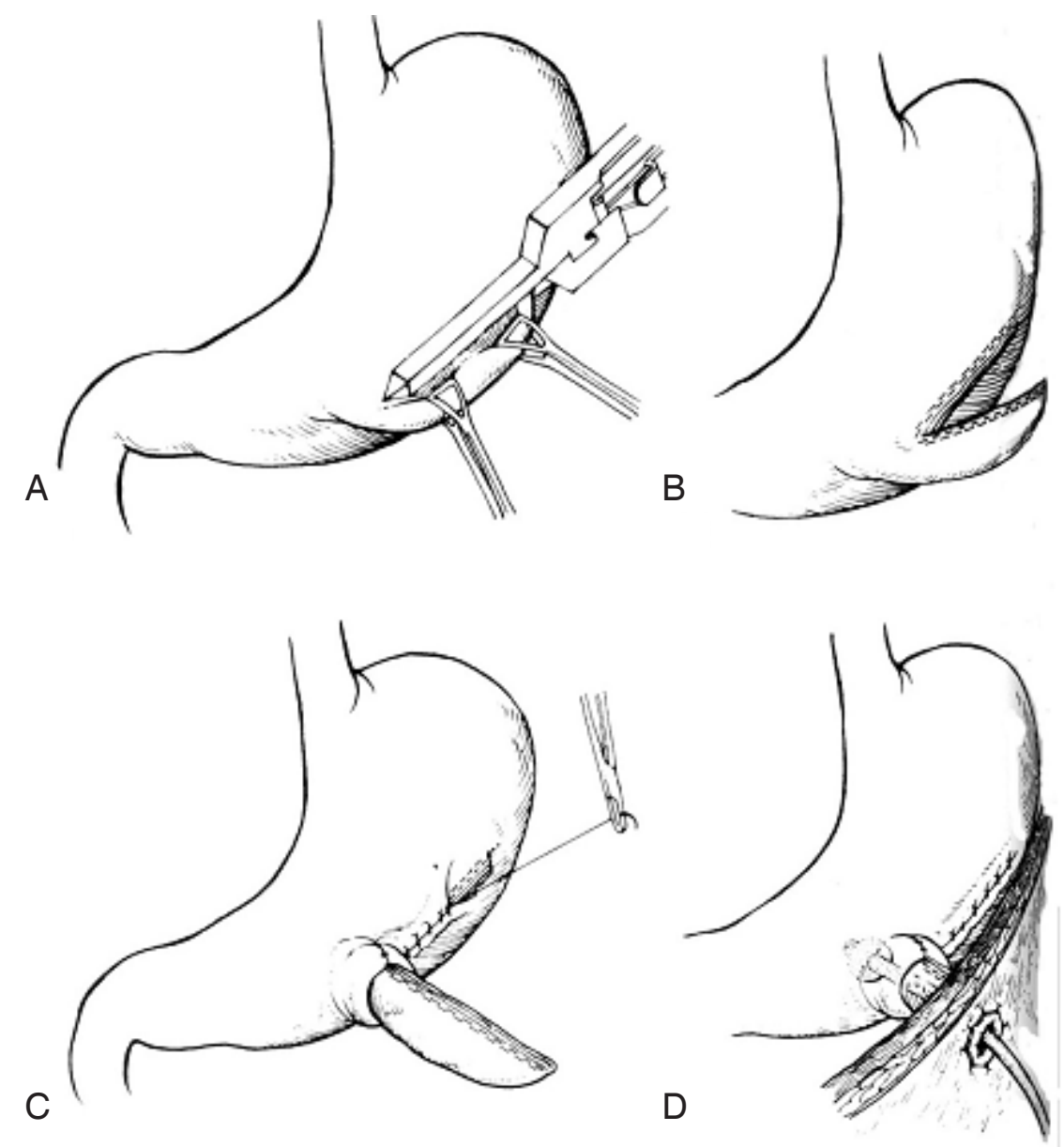

Figura 4: Gastrostomia permanente realizada com grampeador: A) Preensão do estômago e grampeamento ao longo da curvatura maior. (B e C) Preparação de um tubo gástrico. D) Aproximação da mucosa com a pele e introdução da sonda. Extraído de Jesseph MJ. Open Gastrostomy. In Fischer EJ. Mastery of Surgery. Philadelphia. Lippincott Williams \& Williams ed, 2007; 69: 839-42.

\section{Tabela 1}

Situações clínicas mais frequentemente associadas à colocação de GEP ${ }^{12}$

Acidente vascular encefálico

Doença do neurônio motor

Esclerose múltipla

Doença de Parkinson

Paralisia Cerebral

Doenças demenciais

Traumatismo crânio encefálico

Pacientes em tratamento intensivo

Neoplasia de orofaringe

Neoplasia de esôfago

Queimaduras

Fístulas digestivas

Síndrome do intestino curto ses eventos quando comparada com a sondagem nasogástrica. ${ }^{12}$ Em meta-análise recente, a GEP esteve associada a menor incidência de interrupções na alimentação, obstrução ou perda do posicionamento da sonda e não aderência ao tratamento quando comparada com a sondagem nasogástrica, no entanto, não houve diferença significativa em relação ao número de complicações, taxa de mortalidade e incidência de pneumonia. $^{13}$

A taxa de complicações após GEP varia de 8 $30 \%$, sendo que as principais estão citadas na tabela abaixo (Tabela 2). ${ }^{12}$

Em estudo feito na Inglaterra, entre abril de 2002 e março de 2003, pelo National Confidential Enquiry into Patient Outcome and Death (NCEPOD), a taxa de mortalidade em 16.648 pacientes submetidos à GEP, foi de aproximadamente $6 \%$; $43 \%$ na primeira 


\section{Tabela 2}

\section{Complicações associadas à GEP}

Perfuração
Hemorragia
Aspiração
Íleo prolongado
Infecção do sítio de inserção
Lesão visceral (Fígado, intestino delgado, colon ou
baço)
Obstrução gástrica
Erosão do botão de fixação
Migração do tubo da gastrostomia
Peritonite localizada ou generalizada
Vazamento ou infecção periostomia
Fístula gástrica residual
Ulceração gástrica ou da pele
Formação de tecido de granulação periostomia

semana após a realização da gastrostomia. A colocação da GEP foi considerada desnecessária em $19 \%$ dos pacientes, o que leva a concluir que pode haver associação entre a mortalidade encontrada e a condição clínica do paciente. ${ }^{12}$ Esse procedimento está contra indicado em pacientes com coagulopatias não corrigidas, instabilidade hemodinâmica, sepse grave, abdome agudo perfurativo ou outras condições que contraindiquem o exame endoscópico. As contraindicações relativas incluem pacientes com doença sistêmica grave, gastroparesia ou obstrução mecânica ao esvaziamento gástrico, cirurgia gástrica prévia ou outra cirurgia abdominal, ascite e obesidade mórbida. ${ }^{12}$

A gastrostomia endoscópica percutânea pode ser realizada, em regime ambulatorial ou no leito do paciente. O preparo inclui a administração de antibiótico profilático e a sedação leve ou profunda. O procedimento (Figura 5) ${ }^{14}$ envolve: ${ }^{2,3,14}$

- A endoscopia convencional com insuflação do estômago;

- A transiluminação do estômago combinada à palpação digital da parede gástrica com definição do sítio da gastrostomia;

- A assepsia do local selecionado para introdução da sonda de gastrostomia e anestesia local;

- Punção com aspiração concomitante do local mediante seringa acoplada a uma agulha fina contendo um pouco de líquido para certificar-se, por meio da endoscopia, a coincidência do escape aéreo com a extremidade da agulha na luz gástrica;

- Incisão na pele e subcutâneo de aproximadamente $1 \mathrm{~cm}$, com introdução de trocarte até a cavidade gástrica;

- Introdução de fio guia pelo trocarte até o estômago, seguida da sua preensão com pinça endoscópica e exteriorização pela boca;

- Passagem da sonda de gastrostomia de silicone ou poliuretano de 18 a $28 \mathrm{~F}$ orientada pelo fio guia de forma retrógrada ou anterógrada.

- Fixação da sonda e parede gástrica ao peritônio com auxílio de balão (Figura 5.2) sem pressão excessiva para evitar necrose da parede gástrica e à pele por meio do anel externo.

\section{3- Jejunostomia}

A jejunostomia é um procedimento cirúrgico que estabelece o acesso à luz do jejuno proximal através da parede abdominal. As vias de acesso habitualmente empregadas para realização da jejunostomia são: laparotomia, laparoscopia e endoscopia.

\subsection{Indicações de jejunostomia}

\subsubsection{Descompressão digestiva}

A jejunostomia temporária é indicada, ocasionalmente, como complemento de operações abdominais de grande porte nas quais envolvem ressecção gástrica e se prenuncia "íleo adinâmico" pós-operatório prolongado, bem como a ocorrência de fístulas digestivas. Como exemplo, a jejunostomia pode ser realizada em complemento à gastrectomia total, sobretudo em pacientes idosos, com doença pulmonar obstrutiva crônica, psicóticos e agitados, onde se deseja evitar o desconforto e os riscos do emprego de sonda nasoenteral.

\subsubsection{Alimentação}

Temporária: Indicada quando o acesso ao trato digestivo está prejudicado, para recuperação e manutenção do estado nutricional, até que seja restabelecido o trânsito alimentar: estenose cáustica envolvendo esôfago e estômago, e, eventualmente, em pacientes com coma prolongado e passado de gastrectomia.

Definitiva: como terapêutica paliativa em pacientes portadores de neoplasia maligna irressecável do estômago. A jejunostomia definitiva também está indicada em pacientes com a deglutição e o apetite 


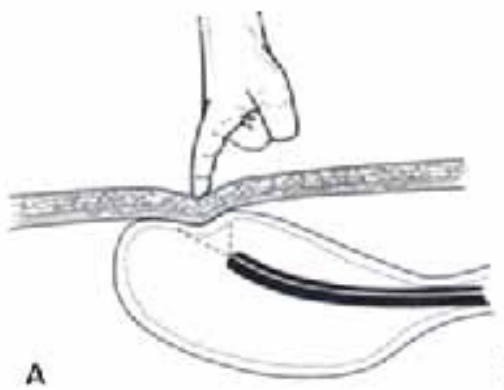

B
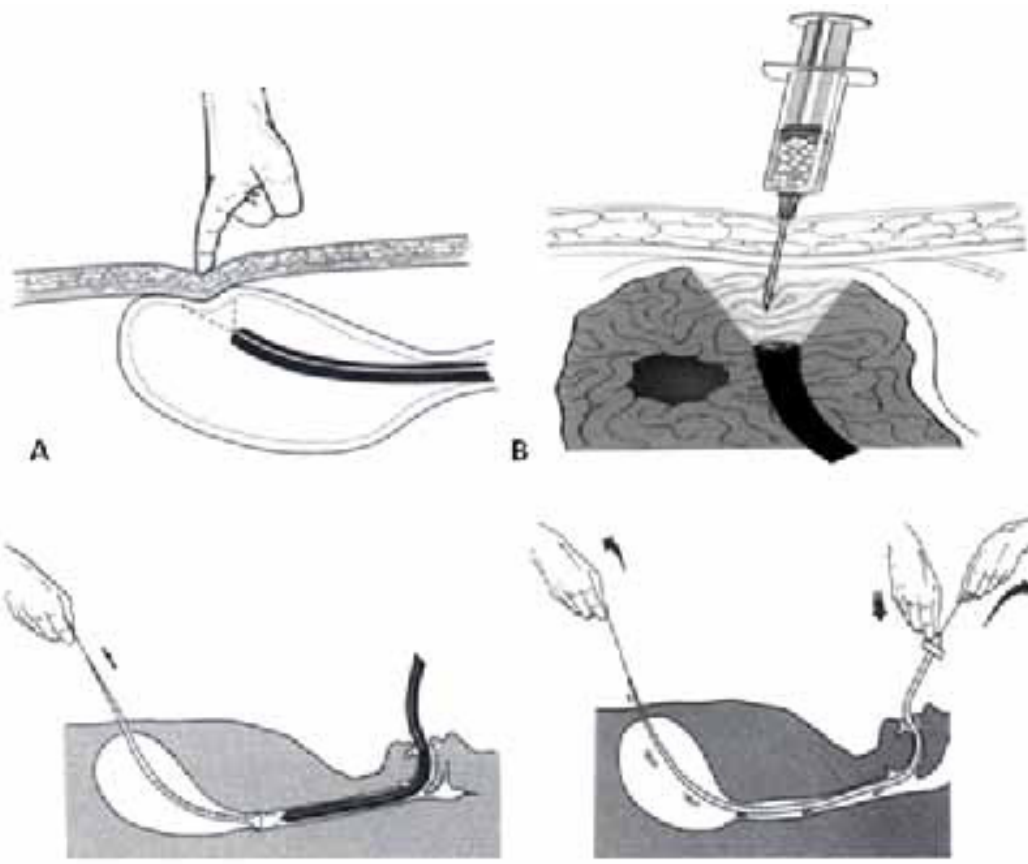

D

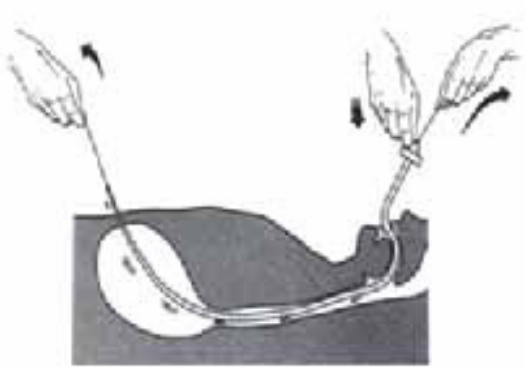

E
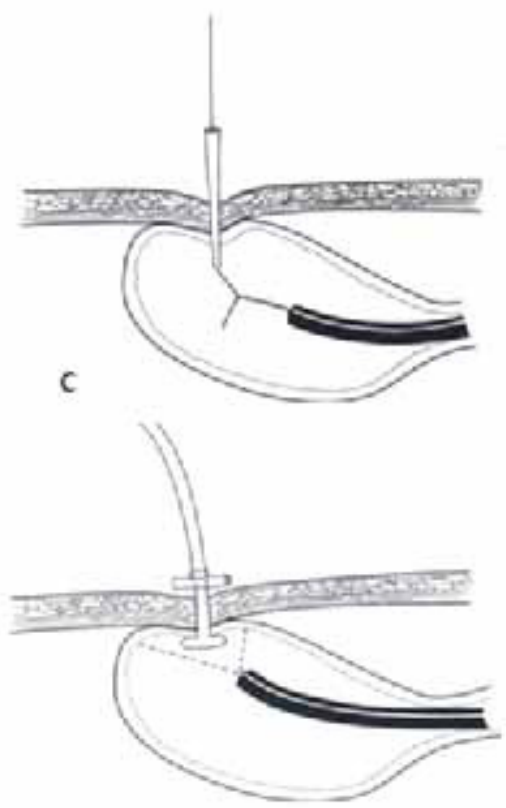

$\mathbf{F}$

Figura 5.1: Gastrostomia endoscópica percutânea: seleção do local de punção por meio da pressão digital da parede abdominal e do estômago com auxílio da transiluminação $(A)$; manobra de segurança (aspiração para certificar-se de que não há outra víscera oca interposta entre o estômago e a parede abdominal) (B); punção e introdução do fio guia(C); exteriorização do fio guia pela boca com o auxílio do endoscópio; (D) fixação da sonda de gastrostomia ao fio guia(E); exteriorização de forma retrógrada e fixação da sonda à parede abdominal $(F)$. Extraído de Jesseph MJ. Open Gastrostomy. In Fischer EJ. Mastery of Surgery. Philadelphia. Lippincott Williams \& Williams ed, 2007; 69: 839-42.
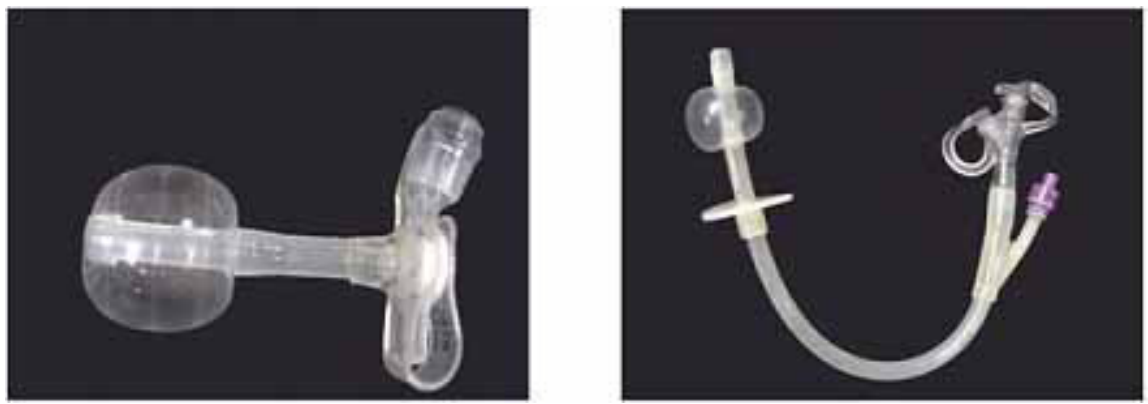

Figura 5.2: Tipos de sonda para gastrostomia percutânea endoscópica com detalhe dos balões de fixação e dos anéis externos.

afetados, como nas doenças neurológicas:- demência, esclerose amiotrófica lateral, seqüelas de acidente vascular cerebral, onde o estômago não pode ser utilizado ou não está disponível (passado de gastrectomia).

\subsubsection{Descompressão e alimentação}

Na dependência da evolução pós-operatória de algumas cirurgias digestivas mais complexas, a jeju- nostomia em determinado momento, pode servir para descompressão do trato digestório e noutra fase ser utilizada para alimentação. Na gastrectomia total por tumor, a jejunostomia no pós-operatório precoce servirá para a descompressão do trato digestório sob efeito do "íleo adinâmico". A anastomose do esôfago com o jejuno tem risco de deiscência que varia de 7 a $13 \%$ na dependência de morbidez associada ${ }^{15}$ e habitual- 
mente um dreno é deixado nas proximidades para monitorar eventuais vazamentos. Na eventualidade de ocorrer uma fístula dirigida para a pele, o dreno abdominal é mantido e resolvido o "íleo adinâmico", iniciase dieta pela jejunostomia, na expectativa de fechamento da fístula no período de até seis semanas.

\section{4- Jejunostomia: aspectos técnicos}

\subsection{Jejunostomia à Witzel ${ }^{16}$ (Figura 6)}

- Incisão mediana supra-umbilical;

- Afastamento cranial do epiploon e do colon transverso com identificação da porção fixa do jejuno, ao nível do ângulo duodenojejunal (ângulo de Treitz);

- Preensão da borda contramesentérica do jejuno proximal com pinças de Babcock, para avaliar a aproximação do jejuno com o peritônio;

- Sutura seromuscular em bolsa na borda contramesentérica;

- Incisão com bisturi ou eletrocautério no centro da sutura, de tamanho apenas suficiente para a colocação de sonda com 8 a 12 F;
- Introdução de sonda de silicone ou borracha na extensão de $20 \mathrm{~cm}$, seguida de fechamento de sutura em bolsa;

- Realização de uma nova sutura seromuscular em bolsa, concêntrica, que é amarrada de maneira a invaginar a sutura anterior, sem provocar estreitamento da luz jejunal;

- Realização de um túnel de 3 a $5 \mathrm{~cm}$ de extensão mediante sutura seromuscular (contínua ou com pontos separados de fio absorvível ou inabsorvível) recobrindo a sonda deitada sobre a parede anterior do jejuno;

- Preensão da linha alba com duas pinças de kocher, exposição do hipocôndrio esquerdo e incisão na pele, de tamanho suficiente para passagem da sonda, na parede anterior do abdômen a $2 \mathrm{~cm}$ da reborda costal pararretal à esquerda;

- Introdução de uma pinça de Schinidt, a partir da incisão da parede abdominal e preensão da sonda colocada no jejuno e um dos fios da sutura em bolsa deixado como reparo para exercer tração sobre o jejuno;
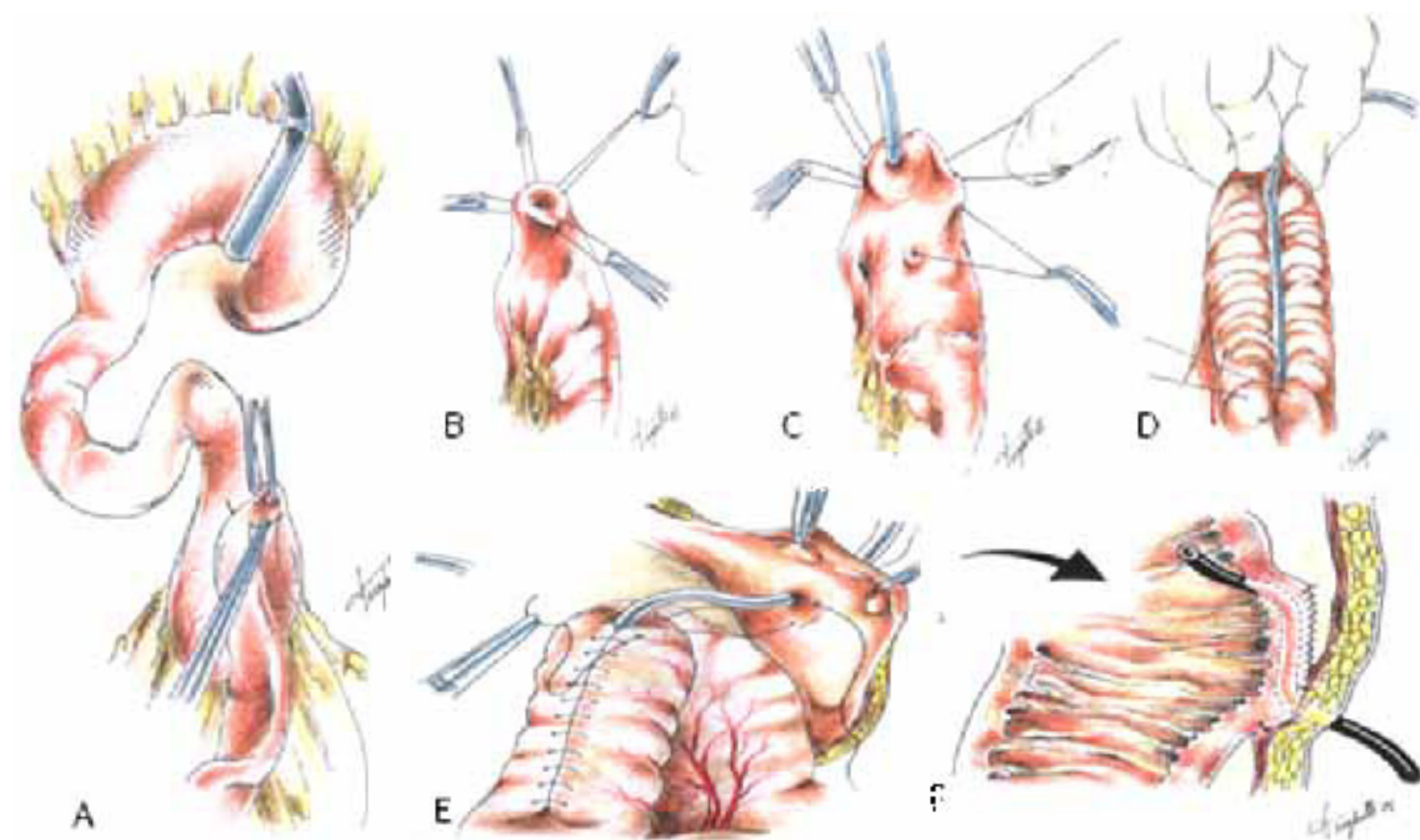

$$
\text { B }
$$

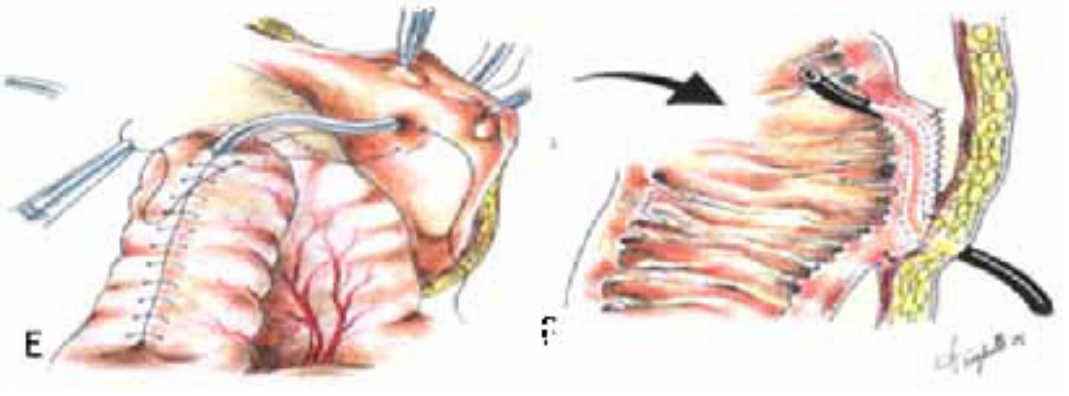

Figura 6: Jejunostomia: seleção do local (A), sutura em bolsa (B), introdução da sonda (C), realização do túnel seromuscular(D) e exteriorização da sonda com fixação do jejuno ao peritônio parietal (E e F). 
- Exteriorização por contra-abertura do conjunto (fio de reparo e sonda) de forma cuidadosa;

- Tração externa com as duas pinças de kocher utilizadas para preensão da linha alba e contra-tração por meio dos dedos indicadores da parede do hipocôndrio esquerdo com o objetivo de aproximar o peritônio parietal da parede jejunal;

- Fixação do jejuno à parede abdominal anterior com pontos cardinais entre seromuscular do jejuno e peritônio parietal, em torno e juntos da sonda;

- Fixação da sonda à pele com ponto de fio inabsorvível;

- Síntese da parede com aproximação da linha alba mediante sutura contínua com fio monofilamentar 1-0 ou 2-0 e da pele com pontos separados de nylon 3-0.

\subsection{Gastrojejunostomia endoscópica percutânea}

Trata-se de uma extensão para o jejuno de uma sonda instalada a partir de uma gastrostomia endoscópica percutânea. Habitualmente uma sonda com diâmetro de 8,5 a 12 Fr é introduzida através de uma sonda de gastrostomia percutânea de 24 a 28 Fr e, por meio de endoscopia ou de um fio guia é conduzida até o jejuno. Esse procedimento está indicado nos pacientes que não toleram alimentação pela gastrostomia ou que apresentam risco elevado de aspiração. ${ }^{17,18}$

\subsection{Jejunostomia endoscópica percutânea}

É um procedimento similar à gastrostomia percutânea endoscópica indicado para pacientes que necessitam de suporte nutricional definitivo e não possuem estômago ou não toleram a alimentação por gastrostomia. Por meio de um enteroscópio ou colonoscópio infantil atinge se o jejuno e por transiluminação orienta-se a punção percutânea pelo trocarte, onde se introduz um fio guia que é tracionado pelo endoscópio até a boca. Na sequiência, a sonda é fixada ao fio guia e conduzida até o jejuno. A jejunostomia percutânea endoscópica é um procedimento técnico mais complexo e é bem sucedido em cerca de $85 \%$ dos casos. ${ }^{19,20}$

\section{5- Gastrostomia e jejunostomia por laparoscopia}

A gastrostomia e a jejunostomia por via laparoscópica começaram a ser realizadas por volta de 1990 na perspectiva de reduzir a morbidade e o tempo cirúrgico, o que não se confirmou nas observações posteriores. O emprego do acesso laparoscópico para a realização da gastrostomia e a jejunostomia é excepecional e deve ser recomendado nas circunstâncias em que a operação principal esteja sendo executada por essa via e na sua evolução há previsão de paresia prolongada do trato digestório ou necessidade de suporte nutricional enteral.Naquelas situações onde as estomias obtidas por endoscopia estiverem induzindo ao refluxo inaceitável com aspiração pulmonar e pneumonia, o acesso laparoscópico pode ser empregado na perspectiva de colocar a sonda mais distal no jejuno ${ }^{4}$ ou acrescentar à gastrostomia uma operação anti-refluxo que possa minimizar tais complicações.

\section{6- Resultados}

Os estudos controlados demonstram resultados similares entre a gastrostomia cirúrgica e a endoscópica percutânea com redução de custos no acesso endocópico. ${ }^{21,22} \mathrm{O}$ procedimento laparoscópico tem resultados semelhantes aos obtidos pela gastrostomia realizada por laparotomia, mas com tempo operatório mais curto. ${ }^{23} \mathrm{O}$ preparo adequado com hidratação, os cuidados com assepsia e antissepsia e a antibioticoterapia profilática podem reduzir significativamente o índice de complicações.

Há estudos demonstrando vantagens da gastrostomia endoscópica percutânea em relação à gastrostomia por laparotomia, com redução de internação, custos e complicações. ${ }^{24,25}$ A incidência de complicações variou de 6 a $56 \%$ para a gastrostomia por laparotomia e de 2 a $15 \%$ para gastrostomia percutânea. ${ }^{26}$

\section{7- Cuidados pós-operatórios}

Após a realização da gastrostomia temporária, a sonda fica aberta, em drenagem espontânea até o restabelecimento do peristaltismo, quando pode ser clampada e utilizada para reposição hidrica e nutricional; em comparação com a via endovenosa, é o método de escolha, principalmente, nos pacientes idosos. A gastrostomia temporária deve ser mantida por pelo menos 3 a 4 dias para garantir uma vedação peritoneal adequada. Além disso, a sonda não deve ser retirada até que a função alimentar tenha sido restabelecida.

A gastrostomia permanente realizada em virtude de obstrução esofágica pode receber líquidos como água e leite dentro de 24 horas, enquanto continua a hiper-alimentação endovenosa. Os líquidos de valor calórico e vitamínico elevados são acrescentados gra- 
dualmente. Após uma ou mais semanas a sonda pode ser removida para limpeza, mas deve ser imediatamente substituída em virtude da tendência a um rápido fechamento do trajeto.

\section{8- Complicações}

A mortalidade após a realização da gastrostomia e jejunotomia é rara se o paciente é adequadamente preparado, se a técnica operatória é bem executada e sem contaminação. A gastrostomia por si só predispõe ao refluxo gastroesofágico, a aspiração e a pneumonia. Desta forma, a indicação de jejunostomia ou da associação de valva anti-refluxo à gastrostomia em pacientes comatosos está bem fundamentada. ${ }^{1}$

A morbidez está relacionada principalmente com a incontinência da estomia, infecção da parede e troca de sondas. Na incontinência, o refluxo da secreção gástrica em torno da sonda é fator de contaminação e infecção peritoneal e parietal, além de lesões cutâneas.

Esses procedimentos, embora de execução simples e rápida, não devem ser subestimados. Em virtude do desconforto que traz e por constituir alternativa de entrada dos alimentos, os pacientes a têm como uma doença e um transtorno e por isso qualquer complicação é muito mal recebida. Estoma pequeno e alto no estômago permite troca fácil da sonda e menor possibilidade de incontinência. A sonda não deve, em princípio, sair pela incisão da laparotomia, mas por contra-abertura pequena, trans ou pararretal esquerda, afastada da linha mediana para não expor a incisão cirúrgica à contaminação e à supuração, com possibilidade de evisceração e, tardiamente, de hérnia incisional. ${ }^{1}$

\section{9- Aspectos éticos e legais do em- prego da gastrostomia e jejunos- tomia para suporte nutricional paliativo}

A velocidade da transição demográfica, no Brasil, foi rápida. Houve envelhecimento da população em virtude da diminuição da fecundidade e do aumento da expectativa de vida. A proporção de idosos com mais de 65 anos que era de 2,7\% em 1960 passou para 5,4\% em 2000 e no ano de 2050 deverá superar a de jovens, alcançando 19\%. Acompanhando a transição demográfica, verificou-se a transição epidemiológica; as doenças infecciosas e parasitárias que lideravam as causas de morte cederam lugar para as afecções crônicas e degenerativas. ${ }^{27}$

Nesse contexto, o aumento da população com doenças neurológicas de evolução progressiva (esclerose amiotrófica, doença de Alzheimer, doença de Parkinson, demência), sequielas de isquemia cerebral e neoplasias avançadas e o desenvolvimento de procedimentos cirúrgicos menos invasivos ampliou a discussão sobre os limites da indicação de suporte nutricional como cuidado paliativo, especialmente por meio da gastrostomia endoscópica. ${ }^{28}$ Dessa forma, os médicos precisam subsidiar a decisão dos pacientes e familiares, com base na oferta de orientações sustentadas na evidência cientifica e nos princípios éticos e legais da autonomia. Por outro lado, a maioria dos estudos para avaliar o impacto na qualidade de vida do suporte nutricional por meio da gastrostomia endoscópica como cuidado paliativo tem fragilidades metodológicas, o que reforça a necessidade de novas investigações em contraponto à indicação de tratamentos com base em valores e crenças pessoais.

\begin{abstract}
A temporary or permanent access to the stomach or jejunum, through a gastrostomy or jejunostomy, is indicated whenever nutritional support or prolonged decompression of the upper alimentary tract is needed. With the introduction of endoscopic gastrostomy, the utilization of these procedures has increased in the last 25 years, specially in patients with progressive neurologic diseases and in those with advanced cancer. This article deals with the conceptual aspects of gastrostomies and jejunostomies, its primary indications, the preferential means of access in different clinical scenarios as well as the technical modalities most frequently used. The management of the stomas, the results and potential complications are also highlighted. Finally, the ethical and legal implications of greater utilization of these procedures in a palliative setting are also discussed.
\end{abstract}

Keywords: Gastrostomy. Endoscopic Gastrostomy. Jejunostomy. Enteral Nutrition. Digestive Decompression. Palliative Care. 


\section{Referências bibliográficas}

1. Jesseph MJ. Open Gastrostomy. In: Fischer EJ. Mastery of Surgery. Ed. Philadelphia: Lippincott Williams \& Williams; 69: 839-42, 2007.

2. Gauderer MWL, PonskY JL, Izant RJ Jr. Gastrostomy without laparotomy: a percutaneous endoscopic technique. J Pediatr Surg. 1980; 15: 872-5.

3. Ponsky JL, Gauderer MWL. Percutaneous endoscopic gastrostomy: a nonoperative technique for feeding gastrostomy. Gastrointest Endosc. 1981; 27: 9-11.

4. Rosser JC Jr, Rodas EB, Blancaflor J, Prosst RL, Rosser LE, Salem RR. A simplified technique for laparoscopic jejunostomy and gastrostomy tube placement. Am J Surg. 1999; 177: $61-5$

5. Oishi H, Shindo H, Shirotani N, Kameoka S. The method of gastrointestinal drainage with a long tube: developed for percutaneoustrans-esophageal gastro-tubing. JPN J Operation. 2002; 56: 193-9.

6. Mackey R, Chand B, MD, Oishi H, Kameoka SL, Ponsky JL. Percutaneous transesophageal gastrostomy tube for decompression of malignant obstruction: report of the first case and our series in the US. J Am Coll Surg. 2005; 201: 695700.

7. Räty S, Sand J, Lantto E, Nordback I, Postoperative acute pancreatitis as a major determinant of postoperative delayed gastric emptying after pancreaticoduodenectomy. J Gastrointest Surg. 2006; 10: 1131-9.

8. Jimenez RE, Fernandez-Del Castillo C, Rattner DW, Warshaw AL. Pylorus- preserving pancreaticoduodenectomy in the treatment of chronic pancreatitis. World J Surg. 2003; 27: 1211-6.

9. Capella JF, Capella R. Gastro-gastric fistulas and marginal ulcers in gastric bypass procedure for weight reduction. Obes Surg. 1999; 9: 22-7.

10. Fobi M, Lee H, Holness R, DeGaulle C. Gastric bypass operation for obesity. World J Surg. 1998; 22: 925-35.

11. Ceneviva, R; Yamasaki, R; Donahne, P.E.; Pinto Coelho, J.R.G. Operações sobre o Estômago e Duodeno - Gastrostomia. In: Coelho J. Aparelho Digestivo - Clínica e Cirurgia. Rio de Janeiro: Medsi ed; 50: 329-331, 1990.

12 Kurien M, McAlindon ME, Westaby D, Sanders DS. Percutaneous endoscopic gastrostomy (PEG) feeding. BMJ. 2010 May 7;340:c2414.

13 Gomes CA Jr, Lustosa SA, Matos D, Andriolo RB, Waisberg DR, Waisberg J. Percutaneous endoscopic gastrostomy versus nasogastric tube feeding for adults with swallowing disturbances. Cochrane Database Syst Rev. 2010 Nov 10;(11): CD 008096
14 Ponsky JL. Percutaneous endoscopic gastrostomy. In: Fischer EJ. Mastery of Surgery. Philadelphia: Lippincott Williams \& Williams ed; 70: 843-49, 2007

15. Sauvanet A, Mariette C, Thomas P, Lozac'h P, Segol P, Tiret E, Delpero JR, Collet D, Leborgne J, Pradère B, Bourgeon A, Triboulet JP. Mortality and Morbidity after resection for adenocarcinoma of the gastroesophageal junction: predictive factors. J Am Coll Surg. 2005; 201:253-62.

16. Harbison SP. Intubation of the stomach and small intestine. In: Yeo CJ. Shackelford's surgery of alimentary tract. Philadelphia:Saunders-Elsevier ed; 52: 749-59, 2007.

17. Bumpers HL, Luchette FA, Doerr EL, Hoover EL. A simple technique for insertion of PEJ via PEG. Surg Endosc. 1994; 8: 121-3.

18. Baskin WN. Percutaneous endoscopic gastrostomy and placement of a jejunal extension tube. Tech Gastrointest Endosc. 2001; 3: 30-41.

19. Coates N, Macfadyen B. Endoscopic jejunal access for enteral feeding. Am J Surg. 1995; 169: 627-8.

20. Shike M, Latkany L, Gerdes H, Bloch AS. Direct percutaneous endoscopic jejunostomies for enteral feeding. Gastrointest Endosc. 1996; 44: 536-40.

21. Steigman GV, Goff JS, Silas D. Endoscopic versus operative gastrostomy. Final results of a prospective, randomized trial. Gastrointest Endosc. 1990; 36: 1.

22. Lydiatt DD, Murayama KM, Hollins RR, Thompson JS. Laparoscopic gastrostomy versus open gastrostomy in head and neck cancer patients. Laryngoscope. 1996; 106: 40710.

23. Scott JS, De La Torre RA, Unger SW. Comparison of operative versus percutaneous endoscopic gastrostomy tube placement in the elderly. Am Surg. 1991; 57: 338.

24. Gutt CN, Held S, Paolucci V, et al. Experiences with percutaneous endoscopic gastrostomy. World J Surg. 1996; 20(8): 1006-8.

25. Ponsky JL, Gauderer MW, Stellato TA. Percutaneous endoscopic gastrostomy: review of 150 cases. Arch Surg. 1983; 118(8): 913-4.

26. Steigmann GV, Goff JS, Silas D, Pearlman N, Sun J, Norton L. Endoscopic versus operative gastrostomy: final results of a prospective randomized trial. Gastrointest Endosc. 1991; 36: $1-5$.

27 In: Brito, F. A transição demográfica no Brasil: as possibilidades e os desafios para a economia e a sociedade. Belo Horizonte: CEDEPLAR/UFMG, Texto para Discussão 2007, no 318, p.9.

28 McClave SA, Ritchie, CS The role of endoscopically placed feeding or decompression tubes Gastroenterol Clin N Am. 2006; 35: 83-100: 